EPJ Web of Conferences 28, 12040 (2012)

DOI: $10.1051 /$ epjconf/20122812040

(C) Owned by the authors, published by EDP Sciences, 2012

\title{
Muon reconstruction efficiency measurement in the ATLAS experiment
}

\author{
Nicola Orlando ${ }^{1,2, a}$, on behalf of the ATLAS Collaboration \\ 1 INFN, Sezione di Lecce, Italy, \\ 2 Dipartimento di Fisica, Universita del Salento.
}

\begin{abstract}
The measurement of the muon reconstruction efficiency in the ATLAS experiment based on the "tag and probe" method using decay muons from $J / \psi$ and $Z$ resonances is presented. The efficiency measurement is compared to its value as estimated from simulations in order to provide scaling factors to correct the residual mis-modeling of the ATLAS muon identification performance.
\end{abstract}

\section{Introduction}

This paper describes the measurement of the muon identification efficiency as determined by using the so-called "tag and probe" method at the $J / \psi$ and $Z$ resonances; a detailed description of these studies can be found in [1] and [2]. The method allows to select an unbiased sample of muons by searching for an Inner Detector (ID) track ("the probe") that, along with a well reconstructed muon ("the tag") forms a system with invariant mass consistent with a di-muon resonance. By this procedure a sample of low $p_{T}$ probes (from the $J / \psi \rightarrow \mu^{+} \mu^{-}$decay) and high $p_{T}$ probes (coming from $Z \rightarrow \mu^{+} \mu^{-}$decay) are selected independently of the ATLAS Muon Spectrometer (MS) and can be used to measure the efficiency for reconstructing a muon with MS measurement.

Two categories of muons are reconstructed in ATLAS using the Muon Spectrometer data: Combined (CB) muons, that require the reconstruction of consistent tracks in the MS and in the ID, and Segment tagged (ST) muons, that give additional efficiency as they can recover muons, typically of low $p_{T}$, which did not cross enough precision chambers to allow an independent momentum measurement in the MS. The two classes of muons are implemented in two different reconstruction chains, hereafter referred as chain 1 (Staco) and chain 2 (MuId).

At the $J / \psi$ resonance the tag and probe method suffers from a large background from muons originating from light meson decays in flight or from $\mathrm{b}$ and $\mathrm{c}$ semi-leptonic decays identified as tags that, associated to ID tracks, form a system with invariant mass close to the $J / \psi$ mass. By requiring the probe to be associated with an energy deposit in the calorimeter consistent with a minimum ionizing particle, a sample with low background contamination can be selected.

These Calorimeter-tagged probes (CT probes) allow a measurement of the identification efficiency in the MS almost free from the biases induced by the background subtraction procedure. The background contamination in the tag and probe selection at the $Z$ resonance is very low and well understood theoretically, thus allowing for a simple signal yield extraction.

\footnotetext{
a e-mail: nicola.orlando@cern.ch
}

The muon reconstruction efficiency measurement with the $J / \psi$ candidates uses a sample of $p-p$ collisions at $\sqrt{s}=$ $7 \mathrm{TeV}$ collected in 2010 which amounts to $35.5 \mathrm{pb}^{-1}$; the measurement using the $Z$ boson uses a sub-sample of 2011 data corresponding to $193 \mathrm{pb}^{-1}$.

Events were selected on-line by single muon trigger with increased $p_{T}$ threshold during data taking at higher instantaneous luminosity. Good collision events were selected by requiring at least one reconstructed primary vertex with three or more associated ID tracks; more details on quality criteria applied on track selection can be found in [1].

\section{Muon reconstruction efficiency with $J / \psi \rightarrow \mu^{+} \mu^{-}$decays}

Tags are selected among CB muons associated to a good quality ID track and satisfying the following criteria:

- $p_{T}>4 \mathrm{GeV},|\eta|<2.5$

- distance of closest approach to the primary vertex in the transverse plane $\left|d_{0}\right|<0.3 \mathrm{~mm}$ and in the longitudinal coordinate $\left|z_{0}\right|<1.5 \mathrm{~mm}$. Distance of closest approach significances $\left|d_{0}\right| / \sigma\left(d_{0}\right)<3$ and $\left|z_{0}\right| / \sigma\left(z_{0}\right)<3$; - matching with the muon triggering the event.

For each tag in the event, probes are selected as any good ID track with

- $p>3 \mathrm{GeV},|\eta|<2.5$;

- the probe and the tag tracks can be refitted to a common vertex with $\chi^{2} /$ ndof $<6$;

- distance between tag and probe $\Delta R=\sqrt{(\Delta \phi)^{2}+(\Delta \eta)^{2}}<$ 3.5 ;

- requested opposite charge tag and probe pairs falling in the invariant mass window between $2 \mathrm{GeV}$ to $3.6 \mathrm{GeV}$

In the high multiplicity of LHC events, an ID track can pass all the selection criteria in association with more than one tag in the event. In such cases, in order to avoid testing the muon reconstruction result more than once for the same probe, a unique probe-to-tag association is chosen based on the minimum $\chi^{2}$ of common vertex fit. Furthermore, 


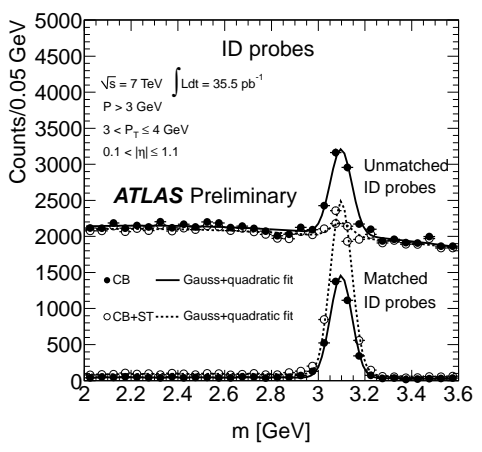

Fig. 1. Invariant mass of the unmatched (upper points) and matched (lower points) tag and probe pairs for $\mathrm{CB}$ (filled circles) and $\mathrm{CB}+\mathrm{ST}$ (empty circles) muons of chain 2 for $0.1<|\eta|<1.1$ and $3 \mathrm{GeV}<p_{T}<4 \mathrm{GeV}$ [3]. The curves show the fits described in the text.

in order to prevent an enhanced probability of reconstructing probes in events topologies with nearby tag and probe tracks we request $\eta_{\text {tag }}-\eta_{\text {probe }}>0.5$ or $\phi_{\text {tag }}-\phi_{\text {probe }}>0.22$.

The tag and probe pairs were divided into two categories, those in which the probe was reconstructed as a muon (matched) and those in which the probe was not reconstructed as muon (unmatched). Figure 1 shows the invariant mass distribution for a sub-sample of the selected tag and probe pairs. The mass distribution is shown separately for probes classified as matched and unmatched with respect to $\mathrm{CB}$ and to $\mathrm{CB}+\mathrm{ST}$ chain 2 muons. The distribution of matched probes shows a clean peak at the $J / \psi$ mass with low background while the distribution of unmatched probes has a peak on top of a large background.

The reconstruction efficiency was obtained as the ratio of the number of events in the peak of the matched distribution to the total number of events in the two mass peaks. A binned maximum log-likelihood fit was performed simultaneously on the two distributions, with the following parametrizations:

$$
\begin{aligned}
\text { Matched } f_{M}(m) & =N_{\text {tot }} \epsilon G\left(m ; \mu_{M}, \sigma_{M}\right)+P_{M}(m) \\
\text { Unmatched } f_{U}(m) & =N_{\text {tot }}(1-\epsilon) G\left(m ; \mu_{U}, \sigma_{U}\right)+P_{U}(m)
\end{aligned}
$$

where $G(m ; \mu, \sigma)$ is a Gaussian distribution with mean $\mu$ and standard deviation $\sigma$, used to describe the signal peak, and $P$ is a polynomial function used to describe the background. The main parameters extracted from the fit are the number of tag and probe pairs in the signal peak $N_{\text {tot }}$ and the reconstruction efficiency $\epsilon$. The mean and width of the two Gaussian distributions were forced to be the same and second-order polynomials were used for the background shape modeling.

A number of checks have been performed to study the dependence of the results on analysis details in order to assess the underlying systematic uncertainties affecting the measurement: these are mainly related to background and signal shape as well as the fitting method [1]. The Figure 2 shows the efficiency for chain 2 in the barrel region $(0.1<$ $|\eta|<1.1)$ with respect to ID tracks with $p>3 \mathrm{GeV}$ as a function of the probe $p_{T}$. The simulation describes the data well.

The uncertainty on the reconstruction efficiency measurement described above is dominated by the statistical and systematic contribution from the large background in

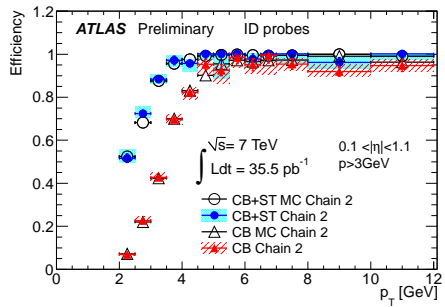

Fig. 2. Efficiency for $\mathrm{CB}$ and $\mathrm{CB}+\mathrm{ST}$ muons of chain 2 as a function of $p_{T}$ in the barrel for data and $\mathrm{MC}$ [3]. The error bars represent the statistical uncertainties while the band around the data points represents the statistical and systematic uncertainties added in quadrature.

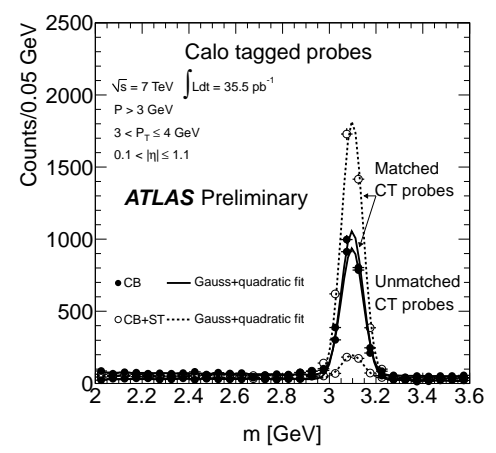

Fig. 3. Invariant mass of the unmatched and matched tag and probe pairs for $\mathrm{CB}$ (filled circles) and $\mathrm{CB}+\mathrm{ST}$ (empty circles) muons of chain 2 for $0.1<|\eta|<1.1$ and $3 \mathrm{GeV}<p_{T}<4 \mathrm{GeV}$ [3]. The probes are selected among calorimeter-tagged muons. The curves show the fits described in the text.

the unmatched sample. The background can be significantly suppressed if the probes are selected among the ID tracks that are identified as CT muons. The calo-tagging algorithm flags ID tracks as calorimeter tagged muons by using the energy deposit in the calorimeter as a discriminant variable. It was optimized to identify muons of $p_{T}$ as low as $2 \mathrm{GeV}$ with good efficiency and purity for the 2010 data taking environment; this allowed to measure the muon reconstruction efficiency with respect to calo-tagged muons in the same kinematic range explored with ID probes. The mass distributions for tag and probe pairs, where the probe is calo-tagged, is shown in Figure 3 for probes matched and unmatched to offline reconstructed muons. The Figure 4 show the efficiency for chain 2 in the barrel region with respect to CT probes with $p>3 \mathrm{GeV}$ as a function of the probe $p_{T}$.

\subsection{Charge dependence}

Due to the toroidal magnetic field of the ATLAS MS, muons with positive (negative) charge are bent towards larger (smaller) $\eta$. At a given $\eta$ for low $p_{T}$ muons there is a strong charge dependency to satisfy the CB track requirements.

However, as long as the ATLAS detector is symmetric with respect to $\eta=0$, the efficiency depends only on $q \times \eta$, where $q$ is the muon charge.

Figure 5 shows the reconstruction efficiency for CB muons of chain 2 as a function of $q \times \eta$ for probes with 


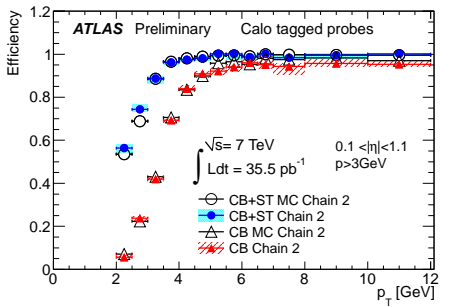

Fig. 4. Efficiency for $\mathrm{CB}$ and $\mathrm{CB}+\mathrm{ST}$ muons of chain 2 with respect to calorimeter-tagged muons as a function of $p_{T}$ in the barrel for data and MC [3]. The error bars represent the statistical uncertainties while the band around the data points represents the statistical and systematic uncertainties added in quadrature.

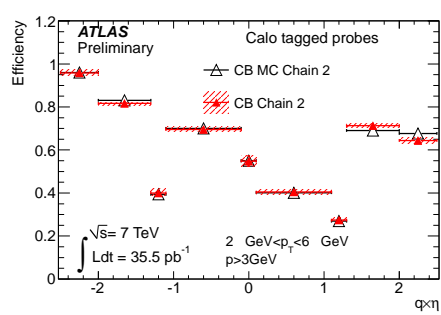

Fig. 5. Efficiency for $\mathrm{CB}$ muons of chain 2 as a function of $q \times \eta$ for data and $\mathrm{MC}$ for the $p_{T}$ range $2 \mathrm{GeV}<p_{T}<6 \mathrm{GeV}$ [3]. Statistical uncertainties are added in quadrature to the systematic uncertainties.

$2 \mathrm{GeV}<p_{T}<6 \mathrm{GeV}$. A strong asymmetry between positive and negative $q \times \eta$ is observed for CB muons. The dependence is well reproduced in the simulation.

\section{Muon reconstruction efficiency with $Z \rightarrow \mu^{+} \mu^{-}$decays}

The clean signature of the $Z \rightarrow \mu^{+} \mu^{-}$decay provides an ideal sample for the tag and probe method at high $p_{T}$. The tag is a CB muon with an associated good quality ID track passing the following selection cuts:

- $p_{T}>20 \mathrm{GeV},|\eta|<2.4$;

- longitudinal distance of closest approach to the primary vertex $\left|z_{0}\right|<10 \mathrm{~mm}$;

- matching with the muon triggering the event;

- isolation [2]: the algorithm establishes that the candidate tag is isolated if

$$
\sum p_{T}^{\Delta R<0.4}(\text { track }) / p_{T}(\text { tag })<0.2,
$$

where the sum is extended over all the tracks (excluding the tag) inside a cone defined as

$$
\Delta R=\sqrt{\Delta \phi(\text { tag, track })^{2}+\Delta \eta(\text { tag }, \text { track })^{2}}<0.4 .
$$

The probes are high quality ID tracks passing the criteria listed below

- $p_{T}>20 \mathrm{GeV},|\eta|<2.5$;

- longitudinal distance of closest approach to the primary vertex $\left|z_{0}\right|<10 \mathrm{~mm}$;

- isolation, as defined for the tag selection.

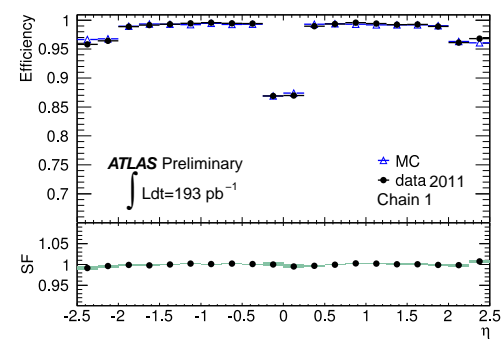

Fig. 6. Efficiency for $\mathrm{CB}+\mathrm{ST}$ muons of chain 1 with respect to the inner tracking efficiency as a function of the pseudorapidity of the muon for muons with $p_{T}>20 \mathrm{GeV}$ [2]. The panel at the bottom shows the ratio between the measured and predicted efficiencies.

The selected tag and probe pairs are required to consist of opposite charge particles, well displaced in azimuth $(\Delta \phi($ tag, probe $)>2)$ and associated to a common interaction vertex; the pairs which fall in an invariant mass window of $10 \mathrm{GeV}$ around the best world average $\mathrm{Z}$ mass value [4] are used for the muon identification efficiency measurement.

The sample purity, as estimated from MC simulations, is $99.3 \%$ with a low contamination from $Z \rightarrow \tau \tau, W \rightarrow \mu \nu$, $W \rightarrow \tau v, c \bar{c}, b \bar{b}$ and $t \bar{t}$. The backgrounds normalizations and shapes are estimated with simulations and subtracted bin by bin from the tag and probe invariant mass distribution.

The integrated luminosity exploited here allows to measure the muon identification efficiency in $p_{T} \times \phi \times \eta$ bins to better match the ATLAS MS geometry layout. An example of the results is shown in Figure 6 for chain $1 \mathrm{CB}+\mathrm{ST}$ muons integrated over $\phi$ and $p_{T}$; excellent agreement is found between data and simulations.

\section{Conclusions}

The tag and probe method at the $J / \psi$ and $Z$ resonances provides a powerful technique for high precision muon identification efficiency measurement over the full ATLAS detector acceptance; the agreement between data and MC is remarkable in a large fraction of the allowed phase space for both the reconstruction chains and algorithms. Scaling factors defined as data over MC efficiency ratios are provided to correct the residual mis-modeling of the ATLAS detector performance in order to increase the accuracy of the muon reconstruction effect unfolding as needed by a wide spectrum of physics analysis performed in the ATLAS experiment.

\section{References}

1. The ATLAS Collaboration, ATLAS-CONF-2011-021, https://cdsweb.cern.ch/record/1336750 (2011).

2. The ATLAS Collaboration, ATLAS-CONF-2011-063, https://cdsweb.cern.ch/record/1345743 (2011).

3. https://twiki.cern.ch/twiki/pub/AtlasPublic/ MuonPerformancePublicPlots/ATL-COM-MUON2011-033.pdf.

4. K. Nakamura et al. (Particle Data Group), J. Phys. G 37, (2010) 075021. 